\title{
Changing mutational patterns
}

UCD is
mechanistically
linked to
improved
response
to immune
checkpoint
inhibitors

The urea cycle (UC) normally functions in the liver to convert excess nitrogen into urea for excretion. However, cells in other parts of the body express various UC enzymes as necessary to provide required metabolic intermediates. Previous studies have shown that some of these enzymes have altered expression in tumours, thereby leading to dependence on UCderived nitrogen sources. This led Lee, Adler et al. to hypothesize that UC dysregulation (UCD) might be widespread in cancer and provide cells with a metabolic advantage.

The authors first extended the previous findings and showed that specific changes in the expression of various UC enzymes can increase proliferation of many types of cancerous and non-cancerous cells. This increased proliferation is connected to increased production of pyrimidine nucleotides owing to the diversion of UC intermediates to the multifunctional enzyme CAD (that has carbamoylphosphate synthetase, aspartate transcarbamylase and dihydroorotase

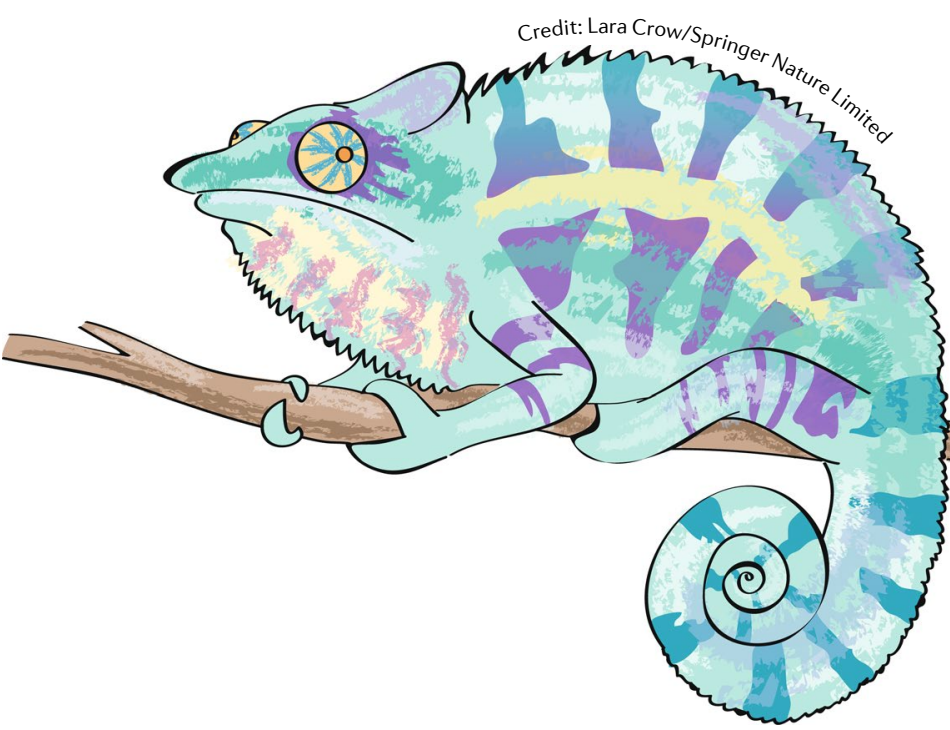

activities), which catalyses the first three steps of pyrimidine synthesis.

Gene expression data from The Cancer Genome Atlas (TCGA) indicated that most tumour types have alterations in UC enzymes that would be expected to promote UCD and increase CAD activity. Furthermore, increased UCD was associated with reduced patient survival in a pan-cancer analysis.

To determine the downstream consequences of UCD, the authors examined pyrimidine levels in mouse and human tumours and in biofluids of cancer patients and mice bearing tumours. Various tumour types exhibited increased pyrimidine:purine ratios. Furthermore, men with prostate cancer had increased urinary pyrimidine levels compared with controls, as did mice with breast or colon tumours. Consistent with this observation, patients or mice with various types of tumours also had reduced plasma urea levels, suggesting that instead of secreting nitrogen as urea, nitrogen was being used in pyrimidine synthesis.

Changes in the pyrimidine:purine nucleotide ratio have been associated with increased mutagenesis; indeed, genomes of tumours with UCD had a significant increase in purine to pyrimidine mutations compared with pyrimidine to purine mutations.

This pyrimidine-rich transversion mutational bias (PTMB) increases over time in cell lines following UCD induction. In patients, however, PTMB is independent of overall mutational load, but is prevalent in many tumour types and is significantly associated with UCD. Furthermore, PTMB-derived changes in the DNA were also detected in mRNAs and proteins.
Although a link between a high mutational load and response to checkpoint inhibitor immunotherapy has been reported, additional studies have found that tumours with the same overall mutational load respond differently to such inhibitors. Therefore, the authors analysed the connection of UCD and PTMB to immune checkpoint inhibitor response. Patients with melanoma who responded to inhibitors of either programmed cell death protein 1 (PD1) or cytotoxic T lymphocyteassociated antigen 4 (CTLA4) had tumours with significantly higher UCD levels compared with nonresponders. In addition, mouse colon tumours in which UCD was induced were more responsive to PD1 inhibition than were non-UCD control tumours. Interestingly, various lines of evidence suggested that cells with high UCD might be more immunogenic as a result of purine to pyrimidine mutations that produce more hydrophobic amino acids. This in turn creates peptide neoantigens that are predicted to have increased major histocompatibility complex (MHC) class I binding, leading to increased $\mathrm{T}$ cell activation.

Overall, these results indicate the widespread nature of UCD in cancer, and suggest that UCD is mechanistically linked to improved response to immune checkpoint inhibitors. Furthermore, the changes in UC-related metabolites in urine and plasma could potentially be used as biomarkers to improve early detection of cancer.

Sarah Seton-Rogers

ORIGINAL ARTICLE Lee, J. S., Adler, L. et al. Urea cycle dysregulation generates clinically relevant genomic and biochemical signatures. Cell 174, 1559-1570 (2018)

FURTHER READING Keshet, R. et al. Rewiring urea cycle metabolism in cancer to support anabolism. Nat. Rev. Cancer https://doi.org/ 10.1038/s41568-018-0054-z (2018) 